\title{
2 CAM AT STANDSTILL AND IN ERUPTION
}

\author{
Alan L. Kiplinger \\ Department of Astronomy \\ haverford College
}

\begin{abstract}
Absolute spectrophotometric observations of the prototype diiarf nova $Z$ Cam have been obtained at standstill and during the course of an eruption. Optical continuum slopes during the final stages of the rise to maximum appear steeper (bluer) than those observed during decline and at standstill. The Balmer jump is observed to be in shallow absorption during decline and in deeper absorption during the rise to maximum and at standstill. A composite energy distribution constructed for $Z$ Cam at standstill is compared with fluxes derived from steady state ( $\alpha$-mode 1 ) accretion disk models which utilize a grid of stellar energy distributions to synthesize the disk fluxes. The riost reasonable accretion disk fit to the observations at standstill suggests a mass transfer rate of $\sim 3 \times 10^{17} \mathrm{~g} \mathrm{sec-1}$. The steady state model does not fit the observations of 2 Cam during decline, nor does any arbitrary linear combination of stellar energy distributions. The results suggest that the accretion disk of decline is time dependent and exhibits an emission character which differs from that seen at standstill.
\end{abstract}

